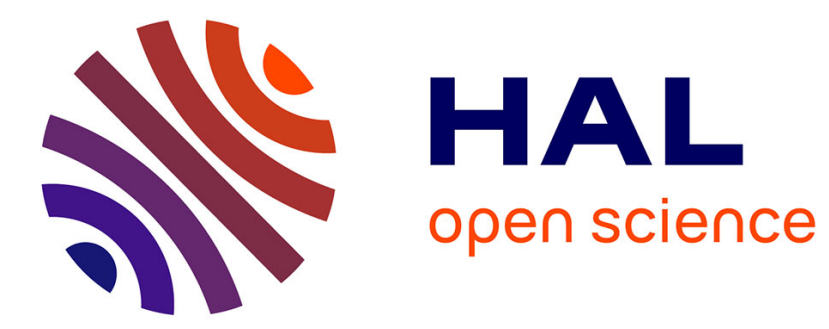

\title{
Les Termes d'adresse dans un bureau parisien
}

Denis Guigo

\section{To cite this version:}

Denis Guigo. Les Termes d'adresse dans un bureau parisien. L'Homme - Revue française d'anthropologie, 1991, 31 (119), pp.41-59. halshs-00470020

\section{HAL Id: halshs-00470020 \\ https://shs.hal.science/halshs-00470020}

Submitted on 3 Apr 2010

HAL is a multi-disciplinary open access archive for the deposit and dissemination of scientific research documents, whether they are published or not. The documents may come from teaching and research institutions in France or abroad, or from public or private research centers.
L'archive ouverte pluridisciplinaire HAL, est destinée au dépôt et à la diffusion de documents scientifiques de niveau recherche, publiés ou non, émanant des établissements d'enseignement et de recherche français ou étrangers, des laboratoires publics ou privés. 


\title{
Persée
}

\author{
http://www.persee.fr
}

\section{Les Termes d'adresse dans un bureau parisien}

\section{Denis Guigo}

Guigo Denis, . Les Termes d'adresse dans un bureau parisien. In: L'Homme, 1991, tome 31 n¹19. pp. 41-59.

Voir l'article en ligne

\begin{abstract}
Avertissement
L'éditeur du site «PERSEE »- le Ministère de la jeunesse, de l'éducation nationale et de la recherche, Direction de l'enseignement supérieur, Sous-direction des bibliothèques et de la documentation - détient la propriété intellectuelle et les droits d'exploitation. A ce titre il est titulaire des droits d'auteur et du droit sui generis du producteur de bases de données sur ce site conformément à la loi n`98-536 du 1 er juillet 1998 relative aux bases de données.
\end{abstract}

Les oeuvres reproduites sur le site «PERSEE » sont protégées par les dispositions générales du Code de la propriété intellectuelle.

Droits et devoirs des utilisateurs

Pour un usage strictement privé, la simple reproduction du contenu de ce site est libre.

Pour un usage scientifique ou pédagogique, à des fins de recherches, d'enseignement ou de communication excluant toute exploitation commerciale, la reproduction et la communication au public du contenu de ce site sont autorisées, sous réserve que celles-ci servent d'illustration, ne soient pas substantielles et ne soient pas expressément limitées (plans ou photographies). La mention Le Ministère de la jeunesse, de l'éducation nationale et de la recherche, Direction de l'enseignement supérieur, Sous-direction des bibliothèques et de la documentation sur chaque reproduction tirée du site est obligatoire ainsi que le nom de la revue et- lorsqu'ils sont indiqués - le nom de l'auteur et la référence du document reproduit.

Toute autre reproduction ou communication au public, intégrale ou substantielle du contenu de ce site, par quelque procédé que ce soit, de l'éditeur original de l'oeuvre, de l'auteur et de ses ayants droit.

La reproduction et l'exploitation des photographies et des plans, y compris à des fins commerciales, doivent être autorisés par l'éditeur du site, Le Ministère de la jeunesse, de l'éducation nationale et de la recherche, Direction de l'enseignement supérieur, Sous-direction des bibliothèques et de la documentation (voir http://www.sup.adc.education.fr/bib/ ). La source et les crédits devront toujours être mentionnés. 


\author{
DENIS GUIGO
}

\title{
Les Termes d'adresse dans un bureau parisien ${ }^{1}$
}

Denis Guigo, Les Termes d'adresse dans un bureau parisien. - Une grande entreprise confie une étude à un ethnologue qui y voit l'occasion de relever le vocabulaire d'adresse : s'adresse-t-on à un collègue par son nom ou par son prénom ? Lui dit-on Tu, Vous, Monsieur ou Madame ? L'analyse des terminologies employées dégage une dynamique de la " bonne distance " dans laquelle opèrent les clivages (sexe, âge) et les principes organisationnels (hiérarchie, division des tâches). L'étude des termes d'adresse ouvre ainsi des pistes pour la construction de l'entreprise comme objet de recherche dans une perspective ethnologique : une entreprise n'est pas un isolat, mais en son sein naissent et évoluent des fonctionnements spécifiques qui renvoient en premier lieu à l'exercice de la différenciation.

Vous me ferez ce que tu peux, Je vous dirai ce que tu veux...

Léo FERré, L'Amour fou.

Me voici en 1985 chargé d'une enquête ethnographique dans une grande entreprise française ${ }^{2}$. Il s'agit de parcourir les usines pendant six mois pour élaborer un rapport sur les nouvelles méthodes de management participatif dans les ateliers ${ }^{3}$. Un jour par semaine environ, je vais voir les commanditaires de l'étude, dans le grand bloc de bureaux caparaçonné de verre et d'acier qui abrite le siège social parisien de la Compagnie. Cet immeuble regroupe auprès de la direction générale plusieurs milliers de « fonctionnels », ainsi nommés par opposition aux « opérationnels » qui produisent dans les usines et transforment la matière. Le grain que les fonctionnels ont à moudre, c'est du papier et de l'information.

Le demandeur est l'une des directions du siège social, forte d'une centaine de personnes. J'en côtoie régulièrement une partie : quarante-cinq personnes dont il va être question ici ${ }^{4}$. L'espace qu'occupent ces hommes et ces femmes est bien délimité : ils sont répartis de part et d'autre d'un austère couloir central, dans une trentaine de bureaux sur les portes desquels figurent discrètement des noms et des sigles, au huitième étage d'une des ailes du grand immeuble. C'est là qu'ils travaillent et pratiquent la sociabilité du bureau : salutations 
rituelles du matin (si on se croise plus tard dans la journée, on se demande : « On s'est vus aujourd'hui ? On s'est pas vus ! », avant de se serrer éventuellement la main), regroupements informels à midi pour aller à la cantine (si Dupont ne mange pas avec ses commensaux habituels, c'est un événement), et tout un ensemble de modalités qui les distinguent les uns des autres et dessinent les facettes de l'identité de chacun.

L'étude m'offrait de nombreuses occasions de fréquenter les membres de la direction. Là naquit l'idée de prêter attention à leur vocabulaire d'adresse et de cerner l'organisation sous-jacente à leurs pratiques. J'ai mis à profit les derniers jours de l'enquête pour interroger séparément une quinzaine d'agents (employés, cadres et chefs de service) sur les termes d'adresse qu'eux-mêmes et leur entourage utilisaient. L'ensemble des réponses m'est apparu suffisamment structuré pour se prêter à l'analyse : même si le matériau n'est pas exhaustif, la variété des positions des informateurs, la connaissance que chacun a du langage de ses voisins et le recoupement des informations fournissent une base cohérente pour décrire le vocabulaire d'adresse et la dynamique de son utilisation ${ }^{5}$.

\section{La hiérarchie fondatrice}

Avant d'examiner les termes d'adresse, présentons les protagonistes. L'observateur ne peut qu'être frappé par l'omniprésence de la dimension hiérarchique. Les bureaux des membres de la direction sont approximativement distribués de la manière suivante : les plus hauts responsables sont " côté rue " avec leur secrétaire, tandis que les autres sont " côté cour ». Par ailleurs, les bureaux d'un chef et de sa secrétaire sont en général entre ceux de deux sous-chefs. La disposition des locaux reflète ainsi grossièrement la pyramide de l'organigramme, cette description officielle par laquelle l'entreprise se donne à voir, et qui permet de saisir en un coup d'œil la division théorique des tâches et la hiérarchie.

L'organigramme se présente comme une imbrication de « râteaux »:

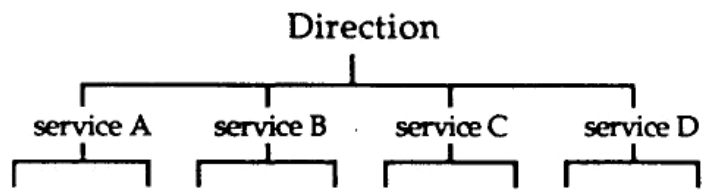

C'est une différenciation à gros grain, car on n'y distingue pas les noms de ceux qui ne sont pas titulaires d'au moins une dent d'un râteau. En revanche, lorsque l'organigramme est « mis à plat » pour se transformer en liste téléphonique, on identifie tous les agents, même les sans grades. Cette transformation met en évidence les cinq niveaux hiérarchiques principaux qui distinguent les membres de la direction, répertoriés chacun selon son rang par 
rapport au directeur : 3 caractères de décalage pour un sous-directeur, 6 pour un chef de service, 9 pour un cadre simple (non chef de service), 12 pour un collaborateur (un non-cadre, alias "étam », abréviation de la catégorie « employés, techniciens et agents de maîtrise » de la convention collective).

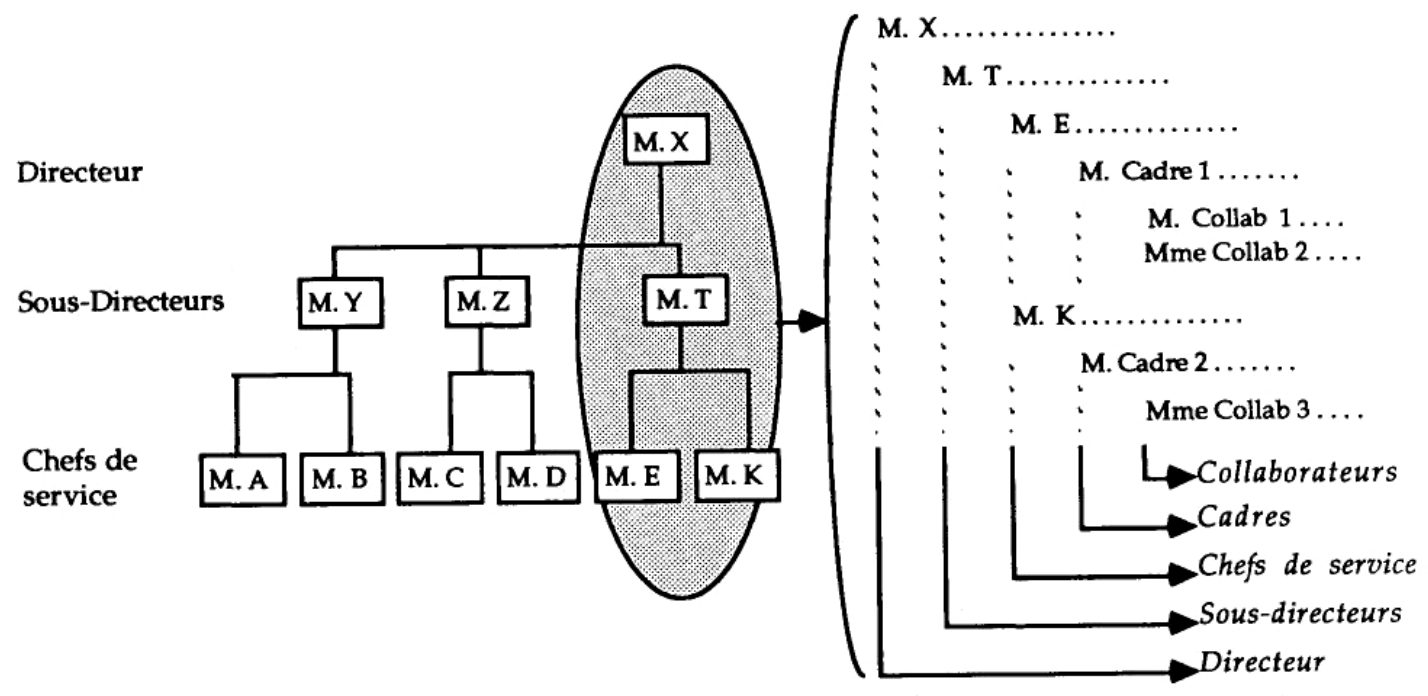

Fig. 1. De l'organigramme à la liste téléphonique

La répartition entre cinq niveaux hiérarchiques principaux génère quatre clivages qui s'emboîtent de manière segmentaire. Le premier sépare les cadres des collaborateurs : ceux-là étant censés encadrer ceux-ci. Cependant, certains cadres sont reconnus comme tels non pas en vertu de leur pouvoir hiérarchique, mais pour leur autorité d'expert : il s'agit en particulier de diplômés de l'enseignement supérieur, qui n'ont parfois aucun subordonné, alors que certains noncadres commandent plusieurs personnes. Par ailleurs, un collaborateur peut être nommé cadre par promotion interne. Il est d'abord « cadre A » (A pour assimilé, cadre imparfait en quelque sorte), puis cadre tout court ; pour certains c'est un " bâton de maréchal ", pour d'autres une étape d'une brillante carrière. " Être ou ne pas être cadre » est donc d'abord une affaire de statut avant de relever de l'autorité exercée. Dans ces bureaux peuplés de " fonctionnels ", il s'agit là de la distinction symbolique entre « ceux d'en haut » et " ceux d'en bas ", distinction qui entraîne un traitement spécifique quant à l'information qu'on leur dispense, l'horaire, la rémunération, le système de prévoyance, etc.

Les autres clivages s'emboîtent au sein de la catégorie des cadres. Les responsables d'un secteur suffisamment important sont nommés chefs de service : ils bénéficient alors d'un bureau individuel, aux cloisons opaques. A partir du rang de sous-directeur, ils disposent d'une voiture de fonction et une secrétaire leur est rattachée. Au-dessus règne le directeur : seuls les sous-directeurs et les chefs de service le rencontrent régulièrement au cours de réunions de travail. 
Les autres doivent saisir leur chance au vol pour évoquer avec lui les sujets qui leur tiennent à cœur sans être accusés de court-circuiter la hiérarchie, car tous les échanges d'information doivent en principe suivre la ligne hiérarchique. Un cadre se souvenait ainsi avec émotion d'une rencontre fortuite aux toilettes : l'intimité d'un lavage de mains dans deux lavabos voisins lui avait permis d'échanger quelques phrases avec le directeur et de voir que le projet qu'il souhaitait lancer serait jugé opportun.

La catégorie des collaborateurs est aussi divisée en sous-ensembles, mais la différenciation se réduit aux échelons de la convention collective (qui déterminent des rémunérations minimales) et n'est donc pas aussi significative que celle qui ordonne les cadres. C'est bien entendu le reflet de la situation d'une " direction fonctionnelle » du siège social, souvent qualifiée d'" armée mexicaine » car les gradés y sont plus nombreux que les sans grades. Chez les « opérationnels » des services de production, la différenciation s'exercerait d'une autre manière, et la catégorie intermédiaire de la "maîtrise " séparerait cadres et ouvriers.

Quant à la vie quotidienne au bureau, nous en noterons quelques traits caractéristiques qui renvoient à la hiérarchie :

- il est exceptionnel qu'un homme soit placé sous les ordres d'une femme ;

- le seul chef de service disposant d'une secrétaire attitrée est le plus âgé d'entre eux (les autres, comme les cadres simples et les collaborateurs, en sont réduits à solliciter les services de la secrétaire du responsable hiérarchique supérieur, mais elle peut avoir des tâches plus urgentes à remplir...) ;

- l'uniformisation du costume est manifeste à mesure qu'on monte en grade $^{6}$;

- les commensaux du repas de midi sont de niveaux hiérarchiques voisins : un jeune cadre ayant pris langue avec un membre de la direction générale dans la file d'attente de la cantine et déjeuné avec lui, ses collègues ne manquèrent pas de le féliciter pour sa rapide "promotion";

- obtenir une promotion est un enjeu essentiel pour la quasi-totalité des agents, et les différents clivages hiérarchiques sont très présents dans les représentations que chacun se fait de ses collègues ;

- le rang de chacun détermine en grande partie le type d'instruments de travail qu'il est censé utiliser. Honte au cadre qui doit faire ses photocopies lui-même faute de secrétaire disponible. Mais le soir, quand le secrétariat est désert, tel haut responsable sera bien embarrassé pour offrir un café ou donner une photocopie à un visiteur tardif. Toucher à une machine à écrire apparâ̂trait à la plupart des cadres comme une déchéance. Même le contact avec le clavier d'un micro-ordinateur pose problème : certains chefs de service brocardaient, avec une ironie qui masquait peut-être une certaine jalousie, un de leurs pairs qui pratiquait l'informatique : "Tiens, voilà notre nouvelle secrétaire ! » Les activités de base du bureau (recevoir un rapport, lire, écrire, signer une note, téléphoner, se réunir...) sont effectuées par chacun selon son rang7. $\mathrm{Au}$-delà des aspects les plus évidents (le collaborateur rassemble l'information 
pour une note, le cadre la rédige, la secrétaire la tape et le chef la signe...), la prégnance de la hiérarchie se manifeste par une multitude de traits significatifs ${ }^{8}$.

Toutefois, le rang de chacun et les attributions correspondant à son niveau hiérarchique ne sont pas définis de manière absolument rigide, car l'organisation doit s'adapter à la diversité des tâches, à leur évolution et aux ressources disponibles : ni les budgets ni les bureaux ne sont facilement extensibles. Un cadre et un collaborateur peuvent exécuter des tâches semblables, mais le cadre est en principe promis à une progression plus rapide. Certains stagnent mais nul n'est renvoyé (sauf pour " faute grave ») ni même rétrogradé : on fait pression sur un indésirảble pour qu'il s'en aille, quitte à négocier une prime de départ.

En définitive, le statut hiérarchique d'un individu résulte d'une combinaison complexe où la dimension temporelle est importante. Au cours de sa carrière, une personne peut être appelée à franchir plusieurs barrières hiérarchiques. Certains jouissent d'avantages considérables au démarrage (notamment les ingénieurs issus de la même « grande école » que le directeur), mais le résultat final n'est jamais vraiment donné d'avance : au sein de la direction, un titulaire d'un simple CAP (certificat d'aptitude professionnelle) — mais qui a à son actif une expérience réussie de commandement en usine - est le chef d'un diplômé d'études supérieures ${ }^{9}$. C'est en quelque sorte en termes de paris que le service du personnel gère les cadres et les collaborateurs, en leur attribuant peu après l'embauche un "potentiel de fin de carrière ». Ce potentiel, modifiable tous les ans, indique le niveau qu'un individu est censé atteindre à 60 ans (pour un cadre, ce sera par exemple : directeur, ou chef de service) et détermine en grande partie la progression de sa rémunération. La hiérarchie - passée, présente et à venir - anime ainsi les différents instruments de gestion de l'entreprise et la trame de son organisation.

Pour représenter la différenciation des membres de la direction, il semble pertinent d'adopter une forme analogue à celle des diagrammes de parenté en ethnologie. Les symboles habituels de la filiation et de la germanité connotent alors la subordination hiérarchique et le fait de relever du même chef (comme dans un organigramme classique), et le symbole traditionnel de la relation d'alliance relie un chef et sa secrétaire. Il est d'ailleurs fréquent qu'une secrétaire suive un responsable dans les différents postes de sa carrière, formant ainsi des paires relativement stables dans l'organisation ${ }^{10}$. La figure 2 représente ainsi les membres de la direction, répartis en cinq niveaux hiérarchiques principaux. On dénombre 25 cadres (dont seulement 2 femmes) et 20 non-cadres (7 employés, 8 employées, 5 secrétaires). 


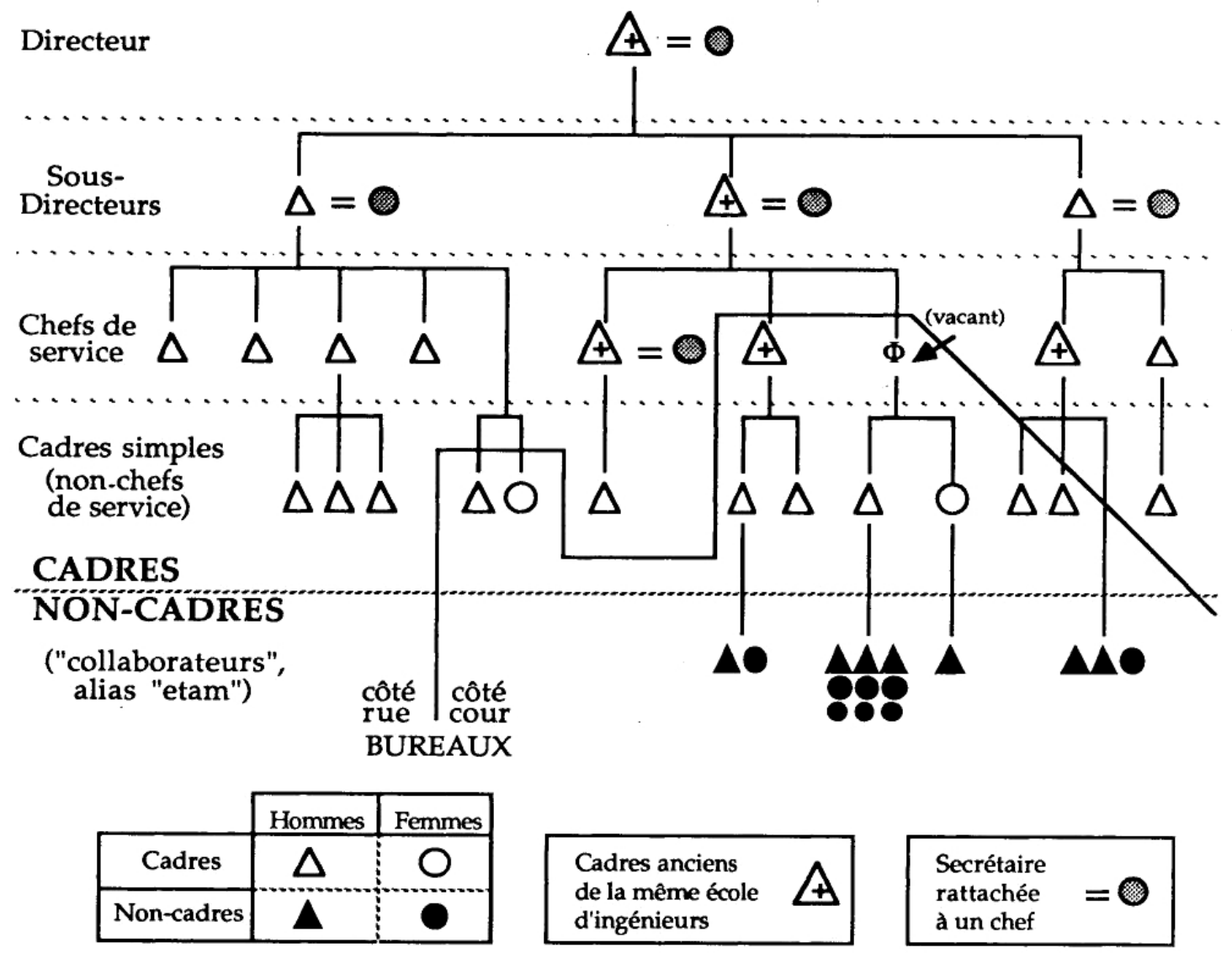

Fig. 2. Les membres de la direction

\section{A Tu et à toi}

Examinons les termes d'adresse du point de vue d'un membre de la direction. Il arrive un beau jour dans un ensemble déjà constitué, où le premier clivage se situe bien entendu entre le $T u$ et le Vous, mais où l'usage - ou l'omission - de Monsieur, de Madame ${ }^{11}$, du nom et du prénom tisse une trame bien réglée. Un cadre parle d'un collègue : "On est trop proches pour que je lui donne du Monsieur, et pas assez pour que je l'appelle par son prénom. » Lors de ses premiers contacts, un nouveau membre répartit progressivement ses interlocuteurs selon différentes catégories d'adresse et les degrés de proximité qu'il ressent. Cette répartition ne se fait pas d'une manière parfaitement consciente ; elle dépend des inclinations du nouvel arrivant, de la façon dont on le reçoit, du « fonds commun » d'appellations en vigueur et des éventuelles relations antérieures avec certains collègues.

Une dizaine de types d'adresse peuvent être relevés au niveau de la direction. La réciprocité est généralement de mise entre deux interlocuteurs : un $T u$ au lieu d'un Vous ne passe pas inaperçu, et le premier à utiliser une nouvelle 
modalité se voit souvent répliquer dans le même registre. Mais certaines relations sont asymétriques : par exemple, un collaborateur salue son chef de service par un « Bonjour Monsieur Frémont ${ }^{12}$ », mais celui-ci répond « Bonjour Moulin, ça va ? ", et dit à sa secrétaire " Mireille, avez-vous reçu les réponses des usines ? " La différence hiérarchique, facteur de distance habituel, rend l'adresse asymétrique. Par ailleurs, le directeur appelle certains cadres par leur nom mais les tutoie : si ces derniers en font autant, c'est qu'ils sont issus de la même école d'ingénieurs que lui ; mais s'ils en restent au Vous, c'est que le directeur les a connus autrefois sur un autre lieu de travail où les chefs tutoyaient paternellement les jeunes cadres.

Lorsque l'adresse entre un subordonné et son chef est symétrique, peuventils se tutoyer ? Pour certains, la familiarité devrait s'étendre à tout le groupe de travail, y compris le chef : " Quand on tutoie, pour moi, c'est qu'il y a une ambiance meilleure " (un collaborateur, 28 ans) ; "Je trouve ridicule que dans une équipe on dise $T u$ à certains et Vous à d'autres " (un cadre, 35 ans) ; "C'est con le vouvoiement parce que ça crée des distances » (un chef de service, 36 ans). Mais dans la plupart des cas, on s'en tient au Vous. Pour un autre chef de service de 36 ans, vouvoyer des collaborateurs est une " marque de considération ». Il ajoute : « C'est plus simple de vouvoyer un subordonné. C'est plus facile de faire passer des messages à caractère hiérarchique si on se vouvoie. " C'est là l'opinion et la pratique habituelles, souvent revendiquées également par " ceux d'en bas ", quand ils affirment préférer le Vous envers le chef : «Je veux garder mes distances. " Le passage à l'état de cadre est en quelque sorte une initiation : certains membres de l'encadrement vouvoient toujours les employés et ne passent au $T u$ que le jour précis où l'un d'entre eux est nommé cadre.

Une alternative importante dans la pratique de l'adresse est de savoir si l'on doit gratifier ou non son interlocuteur d'un Monsieur (ou Madame). On se dispense rarement de le faire dans les cas où une grande différence de niveau hiérarchique ou d'âge sépare les protagonistes, ou bien s'ils ne sont pas du même sexe. Certains critères sont relatifs : dix ans peuvent constituer une grande différence d'âge à 20 ans, peut-être pas à 40 . En outre, une « grande différence " hiérarchique renvoie en général à deux niveaux (sur les cinq de la figure 2), mais le clivage cadre/non-cadre est très marqué, de même que la distance qui sépare, dans l'adresse, un membre de son supérieur direct. Les trois barrières (sexe, âge, hiérarchie) se renforcent mutuellement. Toute familiarité est pratiquement exclue si plusieurs barrières se superposent : on ne voit presque jamais une jeune collaboratrice tutoyer un chef de service d'âge respectable, ni un jeune cadre dire $T u$ à une employée âgée. En revanche, si une seule barrière sépare les protagonistes, une fréquentation suivie peut conduire à de nouvelles modalités d'adresse. Par exemple, un cadre de 31 ans salue d'un "Bonjour Monsieur » les chefs de service qui ont la cinquantaine, mais tutoie et appelle simplement par leur nom certains chefs de service qui ont entre 30 et 40 ans. Il juge impensable de faire l'impasse sur 
le Monsieur quand il s'adresse au sous-directeur (dont le séparent deux niveaux), ou sur le Madame dont il use avec celles qui auraient l'âge d'être sa mère. En revanche, plus de Monsieur ni de Vous envers un pair, qui a 20 ans de plus mais qu'il côtoie quotidiennement : "Gautier, tu vas déjeuner ?"

Lorsque deux protagonistes semblent s'affranchir de barrières qui auraient cantonné d'autres interlocuteurs dans une adresse distante, la raison est extérieure à la situation présente : leur familiarité peut être née hors travail (au football, à la chasse ou au ball-trap) ou dans un autre service (on se tutoie " parce qu'on vient tous deux de S... "). Plusieurs jeunes cadres récemment " sortis du rang " ont un comportement très convivial, dominé par le tutoiement : quand on s'est tutoyé, c'est pour la vie (sauf accident, en particulier lorsque la promotion hiérarchique fait tourner les têtes). Ceux qui montent en grade, passant de collaborateur à cadre dans le même service, cumulent ainsi des familiarités anciennes et nouvelles. Bien entendu, le choix du terme d'adresse entre deux personnes combine toujours créativité individuelle et médiation des usages sociaux préexistants. La combinaison des différents critères n'est jamais donnée d'avance : tel cadre appelle toutes les secrétaires par leur prénom, sauf celle du directeur ; tel autre fait de même pour toutes les secrétaires, sauf pour la sienne.

Le poids des modes d'adresse acquis au fil des ans est considérable. Chacun se forge, consciemment ou non, ses principes d'interaction : " pas de compromission avec les femmes ", " pas de tutoiement avec le chef », affirme tel ou tel. Cela vient en particulier de l'expérience individuelle : l'éducation familiale (« Je vouvoie tout le monde. Ma mère m'a dit : respecte si tu veux être respectée », affirme une employée de 54 ans) ou l'activité antérieure (" J'ai eu du mal à dire Monsieur ; dans l'armée on ne disait jamais Monsieur », confie un cadre de 49 ans, ancien militaire). Mais les déterminations créées dans le travail ne sont pas moins lourdes, et tout " changement d'adresse " entre deux interlocuteurs est significatif. Le rapprochement qui naît du travail en commun produit les changements les plus courants. Si les différences d'âge, de sexe ou de hiérarchie ne s'y opposent pas, le $T u$ peut remplacer le Vous sans stratégie délibérée, encore qu'une explicitation soit parfois jugée nécessaire par l'un ou l'autre : "On se dit $T u$ ? ", " Ça ne te dérange pas que je t'appelle par ton prénom ? », ou bien : "C'est quoi ton prénom ? » Les interlocuteurs règlent ainsi leur adresse à l'heure de la relation.

Les modalités de ce rapprochement dépendent du sexe des protagonistes. Entre hommes, lorsque la combinaison des proximités par l'âge, la hiérarchie et l'interaction du travail fait bannir l'usage de Monsieur, le premier mode d'adresse rappelle le style souvent pratiqué dans l'armée envers les pairs et les inférieurs : "Gautier, vous avez les statistiques des accidents du travail dans les usines ? „ Une plus grande familiarité conduit à remplacer le Vous par le $T u$ en retrouvant ainsi l'adresse traditionnelle du temps des écoliers : "Gautier, tu viens ? » Exceptionnellement, deux hommes vont plus loin et s'appellent par leurs prénoms : c'est que les interlocuteurs partagent une activité de 
loisirs à l'extérieur, connaissent leurs familles respectives ou avaient adopté cet usage auparavant, à la faculté ou dans un autre service. Telles sont donc les trois catégories principales de l'adresse masculine plus familière : le nom suivi de Vous, le nom suivi de $T u$, le prénom suivi de $T u$.

Toutefois, certains trouvent gênant d'apostropher leurs collègues de travail par leur nom de famille. Quand on les tutoie, le malaise provient sans doute du fait que le nom apparaît trop formel pour s'associer au $T u$ : « Je trouve ça aberrant, si on se connaît bien et qu'on se raconte un peu notre vie », dit un cadre, qui n'a pourtant appelé son collègue par son prénom qu'à partir du jour où il l'invita à dîner chez lui. En attendant, le remède est simple : ni nom ni prénom. En cas de nécessité, on use de : « eh, eh ! », " dis donc ! », « s'il te plaît ! ", sans compter les raclements de gorge et les tapes sur le bureau... Avec ceux que l'on vouvoie, il s'agit aussi d'évaluer la distance correcte : on évite le nom quand on ne peut se permettre l'apostrophe de style militaire, tout en estimant que l'interlocuteur ne mérite pas « un Monsieur long comme le bras ». En intercalant ces deux modalités sans nom ni prénom entre les trois catégories décrites ci-dessus, il apparaît donc que les hommes qui délaissent l'usage du Monsieur ont à leur disposition cinq catégories plus ou moins proches : le nom + Vous, le Vous seul, le nom $+T u$, le $T u$ seul, le prénom $+T u$.

Leur « bonne distance » du moment peut chevaucher deux catégories. Par exemple, certains se disent simplement $T u$ mais n'hésitent pas à se héler par leur nom dans le couloir. S'il s'agit d'un changement d'adresse plus fondamental (l'abandon du Monsieur, le passage au $T u$, l'adoption des prénoms), la période transitoire pose problème : «C'est la phase que je déteste le plus, ça m'agace », dit un jeune chef de service qui est entre le $T u$ et le Vous avec un collaborateur d'un autre service. Un cadre remarque que, suivant le terme d'adresse qu'il utilise au téléphone envers son chef (" Frémont ? » ou « Monsieur Frémont ? »), celui-ci lui répond en le tutoyant ou en le vouvoyant. Il est gênant de ne pas savoir où se situer, d'autant que les deux interlocuteurs ne mesurent pas forcément la «bonne distance » de la même façon ("C'est ridicule, mais je n'arrive pas à lui dire $T u$ », dit un cadre à propos d'un collègue qui lui a proposé de se tutoyer). C'est le moment où fleurissent les appellations hors norme, qui expriment le flou de la distance : "Monsieur Lefranc, où en est ton étude ? », " Maître Breuil va bien ? »... Lors de ces périodes de « glissements d'adresse », le contexte (plus ou moins solennel, par exemple) peut faire pencher la balance.

Examinons maintenant le rapprochement dans l'adresse entre femmes de la direction. On ne rencontre jamais l'usage du nom de famille sans un Madame qui le précède. C'est pourquoi le premier terme d'adresse plus familier est le Vous anonyme, sans nom ni prénom. Ensuite deux chemins s'offrent pour une adresse plus proche : soit on adopte le prénom (« Francine, savez-vous ce que Monsieur Guibert m'a dit ? »), soit on passe au Tu tout court. Chacune de ces deux voies peut déboucher sur l'adresse la plus amicale, le prénom suivi du $T u$, et cette dernière modalité, qui demeure exceptionnelle entre hommes, 
se rencontre fréquemment entre interlocuteurs féminins. Cette relative familiarité entre femmes reflète la situation particulière de la direction, où presque toutes sont «collaboratrices ». Ailleurs, la hiérarchie ne manquerait sans doute pas de faire sentir ses effets.

L'adresse entre un homme et une femme utilise le même éventail de catégories qu'entre femmes (de même que l'on n'appelle pas une femme "Durand » mais "Madame Durand ", une femme n'a pas recours au nom seul ${ }^{13}$ ), mais il est rare d'aller au-delà du Vous anonyme. Dans quelques cas, les protagonistes passent au $T u$ ou adoptent le prénom + Vous ; ce dernier style (souvent utilisé par le chef envers sa secrétaire) est appelé « américain » à la direction, lorsqu'il est pratiqué de manière symétrique. Il est censé refléter la convivialité nord-américaine telle qu'on l'imagine (on s'appelle souvent par son prénom dans les firmes américaines). On remarque que ce style « américain » n'est normalement pas utilisé entre hommes : la seule exception est le fait d'un des sousdirecteurs, qui s'adresse ainsi à un de ses cadres « simples » (il n'y a pas de chef de service entre les deux). Mais ce cadre remplit certaines tâches spécifiques de secrétariat : le vocabulaire d'adresse exprime symboliquement son assimilation à une secrétaire. Hors de la présence du sous-directeur, ce cadre aime bien s'y référer par son prénom (« René m'a dit qu'il attend le compte rendu... »), mais nul n'est dupe de cette pratique ludique qui simule une grande proximité14.

Les termes d'adresse utilisés entre femmes ou entre homme et femme peuvent donc aussi être rangés selon le degré de proximité ressenti et le phénomène d'ajustement de l'adresse est donc généralement avéré, mais par des voies qui dépendent du sexe des protagonistes. Il arrive toutefois que plusieurs modalités d'adresse soient pratiquées durablement par les mêmes personnes, avec des variations selon le contexte. Cette variabilité a souvent été évoquée dans la littérature ${ }^{15}$. Dans la direction étudiée, rares sont les relations qui donnent ainsi lieu à deux jeux de rôles différents. Dans tous les cas que j'ai relevés, il existe une différence de niveau hiérarchique entre les protagonistes, et le moins gradé ressent souvent un certain malaise face à ces glissements de vocabulaire. Pas de problème si les contextes sont radicalement différents : on tutoie le chef sur le terrain de football, tandis qu'on maintient le Vous au bureau. En revanche, le jeu de la distanciation variable avec un supérieur peut être mal vécu quand cela change d'une situation de travail à une autre. On rencontre plusieurs cas où un cadre et son chef de service se vouvoient dans l'intimité, mais se disent $T u$ lors de certaines réunions de travail où tout le monde se tutoie. Inversement, un cadre et un chef de service (dont il ne dépend pas) se tutoient seul à seul, mais se disent Vous en présence de tiers. Dans ce type d'interaction, le protagoniste de grade inférieur se sent souvent quelque peu mortifié par la familiarité occasionnelle de son interlocuteur. Il préférerait un vouvoiement constant plutôt que cette camaraderie épisodique, qui rend douloureuses les " remises à distance » après ces rapprochements symboliques.

Jouer du $T u$ et du Vous comme au théâtre est parfois bien vécu. Cela n'est plus du tout le cas si on sent que l'interlocuteur manipule consciemment et 
unilatéralement la distance qu'il vous applique. Plusieurs membres de la direction affirment être déjà irrités par le fait qu'un collègue leur propose un style familier sans pouvoir s'y tenir, même s'il ne s'agit en principe que de lapsus : « Il m'a dit On se tutoie, mais à la sortie d'une réunion il m'a dit Vous. Ça m'a énervé, et maintenant je lui dis Vous ». Quant à la manipulation délibérée de l'adresse, c'est une arme redoutable. Un cadre se remémorait ainsi une réunion de travail où il avait soutenu une position opposée à celle d'un protagoniste de niveau hiérarchique supérieur (mais dont il ne dépendait pas). Alors qu'ils se tutoyaient depuis longtemps, son interlocuteur l'apostropha devant les autres en ces termes : «Mais, Monsieur Després, ne voyez-vous pas que votre solution ne tient pas la route ? » L'interpellé, rendu muet par cette remise à distance, laissa l'autre emporter la décision. Il se jura de ne jamais plus le tutoyer, même si son interlocuteur revenait au $T u$ lors de rencontres ultérieures, ce qui advint effectivement. De la même façon, un autre cadre affirmait avoir utilisé un $T u$ « fait pour empiéter sur le territoire de l'autre » et destiné à influencer ses décisions. On lui avait aussi parfois appliqué ce $T u$ stratégique. Une collaboratrice a évoqué un procédé similaire : «On emploie plus le prénom quand on veut faire suer quelqu'un »; autrement dit, il y a des étreintes qui tuent.

La problématique de la distanciation est donc d'abord un ajustement mutuel entre des protagonistes qui évaluent leur position et le contexte. C'est un processus dynamique, quand par exemple un non-cadre commence à tutoyer son chef « pour se mettre à son niveau » et que ce dernier est « bien obligé de suivre », selon les mots d'un témoin de la scène. Il s'agit là de la redéfinition d'une facette de l'identité individuelle (collaborateur, certes, mais proche des cadres) grâce au positionnement par rapport aux différents « autres ». On rencontre enfin des pratiques de manipulation conscientes, où la distance dans l'adresse est un outil symbolique mis en œuvre par les membres de l'organisation, pour des finalités qui n'ont d'autres limites que celles de leur créativité.

\section{Le système de l'adresse}

La configuration des appellations n'est pas statique : elle évolue sous la pression des nouveaux arrivants (qui sont parfois d'anciennes relations), des particularismes (par exemple, le rite du tutoiement entre anciens de la même école), des pratiques de travail et des stratégies spécifiques d'interaction. Mais le système global de l'adresse, c'est-à-dire les oppositions significatives entre les différentes modalités d'adresse possibles, ne se transforme que lentement. C'est d'abord par rapport à une " synchronie signifiante » que prennent sens les pratiques de chacun.

Récapitulons les différents termes d'adresse en usage à la direction en les classant par ordre de proximité croissante. Tout le monde ne pratique pas toutes les modalités. Selon son histoire personnelle et son expérience dans l'entreprise, chacun répartit ses collègues en deux ou trois catégories, parfois cinq ou six ; 
leur plus ou moins grande proximité étant elle-même liée aux différences de sexe, d'âge et de position hiérarchique. La liste ci-dessous regroupe en huit catégories toutes les adresses utilisées, à l'exception de quelques relations singulières : une interaction totalement distanciée, lorsqu'une personne décide de ne plus adresser la parole à une autre ; une catégorie extrêmement proche, lorsqu'on s'adresse à un collègue par un surnom affectueux ; et quelques cas particuliers où s'installe entre deux individus une modalité spécifique de la " bonne distance " (un cadre âgé dit " Jeune fille, Vous... " à une jeune employée qui s'est mariée, un jeune cadre dis Miss à la seule demoiselle de la direction - qu'il connaît depuis plusieurs années - , un jeune collaborateur dit « Monsieur Hector, Vous... " à un autre assez âgé). Les qualificatifs décrivant certains styles d'adresse découlent directement des expressions utilisées par les membres de la direction : militaire (" comme à l'armée »), américain (" à l'américaine »), amical (« entre amis »). Les désignations des cinq autres styles proviennent de mon interprétation des informations recueillies sur le terrain.

Aux huit catégories renvoient les abréviations M N P V T : M(onsieur ou madame), N(om), P(rénom), V(ous), T(u).

M V Style distant, le plus réservé. Aucun nom n'est indiqué, juste « Monsieur " ou " Madame », d'une politesse impersonnelle.

M N V Style respectueux, presque systématique si les interlocuteurs ne sont pas du même sexe. Quand deux personnes de même sexe sont séparées par une grande différence d'âge ou de hiérarchie, c'est l'adresse la plus courante.

N V Style militaire, pratiqué entre deux hommes d'âges et de grades voi(masc.) sins (même niveau hiérarchique, à la rigueur un seul degré de différence).

V Style poli : une proximité plus accentuée rend un peu gênant d'appeler l'autre par son nom (« Madame » suivi du nom, si c'est une femme), mais la familiarité n'est pas telle qu'elle permette le tutoiement ou l'usage du prénom.

$\mathrm{P} \mathrm{V} \quad \mathrm{Ce}$ style américain ne se rencontre que dans trois situations caractéristiques :

- lorsqu'un chef s'adresse à sa secrétaire, relation éminemment asymétrique puisque celle-ci lui donne du « Monsieur Durand »;

- lorsque deux interlocuteurs de position hiérarchique et d'âge voisins s'apprécient et ont de nombreuses occasions de se côtoyer, mais ne passent pas au tutoiement car ils ne sont pas du même sexe ; - entre femmes, cela marque une relation plus proche que celle du style « poli » ; c'est alors une adresse fréquente dès lors que la position hiérarchique et l'âge sont voisins.

N T Style potache, pratiqué entre hommes, comme à l'école. 
T Style camarade : on est très proche par l'âge et la position hiérarchique et on se rencontre souvent ; on travaille ensemble. Entre hommes, on passe du style « potache " au style " camarade " quand la relation se fait plus intense, lorsque l'usage du nom de famille semble incongru; on pousse difficilement plus loin. En revanche, entre femmes, on va souvent jusqu'à l'usage du prénom.

P T Style amical. Les femmes, sauf les plus âgées, le pratiquent souvent dès lors qu'elles sont de position hiérarchique et d'âge voisins. Entre hommes, des relations hors travail sont indispensables pour dépasser le style « camarade ».

La figure 3 récapitule les différents chemins par lesquels on peut passer d'un degré de proximité à un autre. Les crochets regroupent certains styles, soit parce qu'ils sont à peu près de même niveau (« distant » et « respectueux » par exemple, ou « militaire » et « poli » pour les hommes), soit parce qu'ils constituent deux modalités analogues pour marquer une proximité croissante (pour le rapprochement de deux femmes au-delà du Vous poli, le prénom ou le $T u$ offrent deux voies à peu près équivalentes pouvant déboucher sur le style " amical »). On relève ainsi sur chaque parcours quatre étapes principales ${ }^{16}$, donc trois transitions essentielles (la suppression de Monsieur - ou Madame -, l'adoption du prénom ou du $T u$, l'usage du prénom et du $T u$ ), dont le franchissement est régi par l'opposition de sexe, âge, grade et tâche des protagonistes. Les femmes vont souvent jusqu'au style amical, les hommes en restent plutôt à la camaraderie. Entre homme et femme, on dépasse rarement le style poli.

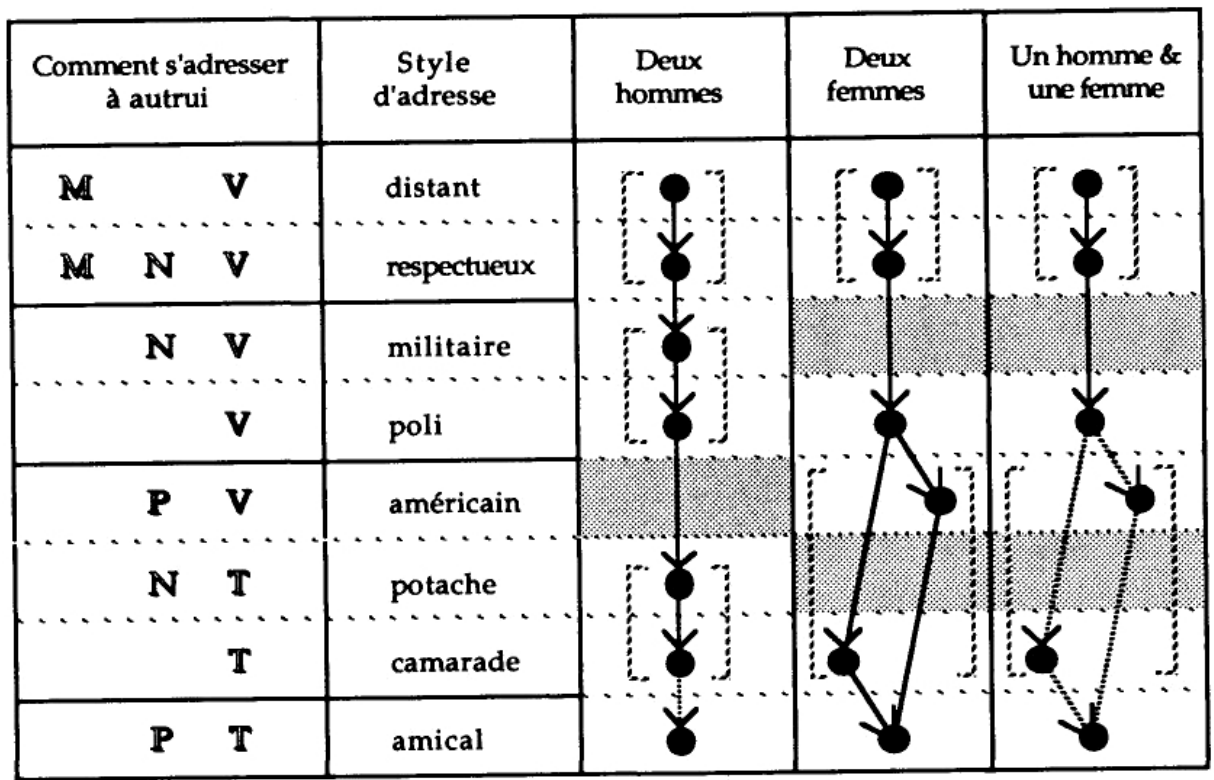

Fig. 3. Les chemins de la familiarité 


\section{La bonne distance}

La distance dans l'adresse peut être qualifiée de "trait structurel »: elle sous-tend le jeu de principes d'opposition qui ne sont pas appliqués de manière rigide, mais ajustés par les protagonistes selon leurs situations respectives et leur histoire. Il y a bien sûr de sévères prédéterminations dans l'entreprise : on est homme ou femme, cadre ou employé... mais l'interprétation des rôles est variable, d'une part parce que les catégories se combinent (le chef est un homme ou une femme), d'autre part parce que l'appartenance à une catégorie est à la fois enjeu et moyen d'action. En outre, le temps des individus est rythmé par la carrière (le changement organisé de catégorie, c'est-à-dire l'évolution d'identité reconnue par l'organisation) et ne coïncide pas avec le temps de l'entreprise, qui court selon d'autres calendriers.

J'ai tenté de décrire la différenciation dans l'adresse, avec sa dynamique particulière : la gestion d'une distance généralement réciproque (Dupont s'adresse à Martin comme Martin à Dupont, sauf biais hiérarchique ou manipulation), non transitive (Jean tutoie Pierre, Pierre tutoie Marcel, mais Jean peut vouvoyer Marcel), évoluant jusqu'à un certain degré de proximité. L'évolution est habituellement irréversible, mais un bouleversement des règles du jeu (promotion hiérarchique, agression...) peut entraîner une « remise à distance ". De même, la distanciation manipulatrice peut provoquer un traumatisme.

Les catégories utilisées par le groupe humain étudié ici ne forment pas une grille universelle. Ailleurs, on rencontre d'autres vocables (" Monsieur le Directeur ", "Collègue »...), et d'autres mises en œuvre des oppositions de sexe, d'âge et de hiérarchie (par exemple, la forme courante de l'adresse polie peut être le vouvoiement, avec Monsieur - ou Madame - suivi du prénom). D'autre part, le clivage sexuel dans la dynamique de l'adresse opérerait sans doute différemment si la haute hiérarchie n'était pas exclusivement masculine ${ }^{17}$. L'agencement des principes d'opposition est donc défini localement et à un moment donné, mais le jeu de la " bonne distance » semble bien être un des outils symboliques fondamentaux des membres des organisations. On retrouve ainsi, mis en situation dans l'entreprise, un concept qui fonde l'analyse de la parenté : ni trop près, ni trop loin (Héritier 1979, 1981 ; Gomes Da Silva 1983) ${ }^{18}$. Cette distanciation est parfois officiellement valorisée, puisque figure dans le dossier d'évaluation de tel ou tel collaborateur cette appréciation flatteuse : " Sait garder ses distances. " A l'opposé, l'adresse syndicale, qui donne uniformément du «Camarade » à chacun, constitue une transgression en quelque sorte incestueuse du point de vue de l'idéologie de l'organisation industrielle hiérarchique $^{19}$.

L'analyse du vocabulaire d'adresse dégage ainsi un fil conducteur pour appréhender de l'entreprise dans une perspective ethnologique : une organisation industrielle apparaît comme un ordre symbolique particulier où s'exercent plusieurs logiques de différenciation, de caractère généralement hiérarchique. 
Les classifications qui sont censées répartir les tâches et les rôles, de même que les finalités officiellement poursuivies par l'entreprise, ne s'imposent certes pas telles quelles à des individus passifs, mais conditionnent dans une certaine mesure le jeu des acteurs : l'organisation assigne un ensemble de médiations à ses membres ${ }^{20}$.

La hiérarchie est à l'œuvre selon des modalités concrètes ou symboliques qui relèvent ou non des normes officielles : en sus de l'analyse de l'organisation du travail, de la gestion du personnel, des pouvoirs exercés et de leur légitimité21, on note en particulier l'espace physique (bureaux, cloisons, téléphones, ordinateurs, ameublement, signalisation...) et le costume (port et couleur des vestes et des cravates, uniformes prescrits ou non...). La hiérarchie et les pratiques différenciatrices qu'elle anime offrent une trame pour articuler plusieurs dimensions de l'organisation, la construire comme objet de recherche et développer une approche comparative. Les membres appliquent consciemment ou non un ensemble de différenciations, il les font évoluer et les manipulent : on saisit ainsi comment une entreprise est conçue (la répartition formelle des rôles et des tâches, et les représentations qu'on en a) en y articulant l'organisation réelle de la vie au travail. L'examen de ces pratiques intéresse l'ethnologue au premier chef, si l'on en croit Claude Lévi-Strauss (1958 : 347) qui souligna l'importance de l'étude des relations entre « ordres conçus » et « ordres vécus ».

En sus de la pratique de la créativité domestiquée que leur propose l'organisation, les membres se livrent en définitive à un bricolage matériel et symboli$q u e$ spécifique ${ }^{22}$. Ils s'en forgent les outils à partir des catégories fondées par l'organisation, mais aussi - en tant qu'êtres totaux, selon l'expression maussienne - en utilisant leurs identités « extérieures » dans la mesure où elles sont mobilisables à l'intérieur. Ni complètement autonome ni totalement réductible aux logiques de la société globale, l'entreprise apparaît bien comme un de ces " groupes qui se perçoivent ou sont perçus comme des unités distinctes » (LeroiGourhan 1968 : 1817), qui caractérisent le champ de l'ethnologie.

Centre de Recherche en Gestion (École Polytechnique)

\& Laboratoire d'Anthropologie urbaine (CNRS), Paris

\section{NOTES}

1. Une première version de cette recherche a été présentée au colloque « Travail et pratiques langagières ", organisé par le réseau multidisciplinaire Langage et travail avec le soutien du PIRTTEMCNRS (Paris, 25-26 avril 1989).

2. Cette entreprise n'a pas pour habitude de faire appel aux services des ethnologues : il faut signaler que j'y ai travaillé autrefois comme ingénieur pendant plusieurs années.

3. Un rapport interne a été remis à l'entreprise. Une publication est parue par ailleurs (GUIGO 1987).

4. Le reste des membres de la direction travaille dans d'autres bâtiments. 
5. Je n'ai pas trouvé, dans la littérature disponible, d'étude ethnographique de la dynamique du vocabulaire d'adresse en entreprise. Voir ACHARD 1982 pour une étude générale du système anthroponymique français, et le numéro XX (4) de L'Homme (1980) pour les formes de nomination en Europe. Voir aussi HYMES, ed., 1964, notamment Roger BROwN \& Marguerite FORD 1964 : 234-244, qui étudient la progression de la familiarité dans l'adresse, et FishmaN, ed., 1968 (en particulier Roger BRown \& Albert GILMAN 1968 : 252-275). L'ouvrage de S. NiEDERMAYER-TAHRI (1978), que M. Pierre Achard m'a aimablement communiqué, présente les termes d'adresse et de référence de trois institutions (une prison, un hôpital, une usine) dans trois pays (France, Allemagne, Angleterre), mais il s'agit d'une approche sociolinguistique centrée sur les termes, qui ne prend pas pour objet le fonctionnement des institutions.

6. Une de mes connaissances, dont l'appartement donnait sur le siège social de la Société, était persuadée que la chemise bleue y était obligatoire en raison du coloris uniforme des silhouettes entrevues aux fenêtres.

7. Voir par exemple le compte rendu de la séance du 30.11.1984 du séminaire « Ethnographie des organisations », où P. Couveinhes analyse l'activité d'un service " fonctionnel » d'une administration.

8. Par exemple, si les deux destinataires d'un rapport sont chefs de service, leurs noms figurent l'un en dessous de l'autre sur la première page, mais s'il s'agit d'un chef et d'un de ses cadres, ils sont indiqués sur la même ligne (MM. Dubois/Durand) pour rappeler que Durand est subordonné à Dubois.

9. Toutefois, la plupart des membres de la direction ignorent que ce chef de service n'a qu'un CAP.

10. Voir à ce sujet Delpeuch \& LAUVERgeon 1986, 1988, et le compte rendu de la séance du 30.11.1984 du séminaire " Ethnographie des organisations " (exposé de P. Couveinhes).

11. Parmi les 15 femmes de la direction, il n'y a qu'une demoiselle (une employée de 32 ans), à l'adresse de laquelle Mademoiselle remplace bien sûr Madame. Ces deux termes fonctionnant de manière identique dans le vocabulaire d'adresse de la direction, je ne les ai pas distingués dans l'analyse.

12. Tous les noms et les prénoms cités sont bien entendu fictifs.

13. Peut-être serait-ce le cas si une femme cadre de la direction commandait un homme plus jeune, mais le seul employé subordonné à l'une d'elle est âgé.

14. L'organisation du vocabulaire de référence est différente de celle de l'adresse. On y relève moins de respect, beaucoup de surnoms cocasses ou dépréciatifs envers les chefs (Pécore pour un chef qui s'exprime d'une manière que certains jugent compassée, Le Che - allusion à Che Guevara - pour un fonceur...).

15. Erving GofFMAN $(1973: 81)$ rapporte par exemple qu'un patron peut appeler sa secrétaire par son prénom lorsqu'ils sont seuls et dire Madame devant un visiteur.

16. Edward T. HALL (1978) analyse l'organisation de l'espace et propose une graduation des distances interpersonnelles, réparties entre quatre « bulles » (publique, sociale, personnelle, intime). La distance conceptualisée par Hall prend en compte la proximité physique ainsi que la vue, le toucher, l'ouie et l'odorat (pour une présentation générale de la proxémique de Hall, voir WinKIN 1981 : 86-91).

17. Georges BaLANDIER (1985) note que dans les sociétés traditionnelles « le pouvoir est nécessairement de principe mâle " (p. 81) et relève que les femmes qui s'en approchent se masculinisent. Il en est manifestement de même dans la quasi-totalité des entreprises industrielles. Certes, il ne s'agit plus alors' d'un pouvoir " par les femmes et sur les femmes » (p..62), mais par et sur les biens de consommation, opérateurs sociaux fondamentaux de la société industrielle. Comment ne pas penser au " principe mâle » lorsque, à l'assemblée annuelle des cadres de la Société, les dirigeants de l'entreprise dissertent longuement sur leur " taux de pénétration » (du marché) ?

18. En conclusion d'une étude sur le système de parenté tibétain, c'est la « distance ou la proximité » qui apparaît comme "le concept de base " (GUIGO 1986 : 113). Faut-il s'étonner de trouver des points communs entre le Tibet et l'entreprise?

19. On sait que la fraternité révolutionnaire, telle que l'entendait le Comité de Salut Public, exigeait le $T u$ envers tous les citoyens.

20. Mary Douglas (1987) examine les manières suivant lesquelles les classifications opérées par les institutions conditionnent la pensée de leurs membres, mais n'utilise pas de matériaux ethnographiques collectés dans les entreprises. Michel BERRY (1983). étudie l'impact des instruments de gestion sur l'évolution des organisations modernes.

21. La variabilité de l'articulation du statut et de l'organisation du travail fournit des pistes pour l'analyse comparative. Par exemple, un individu peut être titulaire d'une sous-direction mais avoir un rang de directeur ou de chef de service. Par ailleurs, alors que la convention collective distingue les cadres 
de « position » 1, 2, 3A, 3B \& 3C (selon les responsabilités assumées), les réunions peuvent s'organiser suivant un critère du genre " tous les $3 \mathrm{C}$ sont invités » ou bien " tous les chefs de service sont invités ».

22. Dans La Pensée sauvage, Claude LÉvi-Strauss (1985) explique que les individus modernes pratiquent la pensée scientifique, pensée domestiquée qui se construit « sur la distinction du contingent et du nécessaire » (p. 35), mais que les mécanismes de la pensée mythique, "cette bricoleuse » (p. 36), ne leur sont pas étrangers, en particulier dans la pratique de l'art. Comme un bricoleur utilisant à des fins imprévues ce qu'il a sous la main, les rites et les mythes " décomposent et recomposent des ensembles événementiels » (p. 49), transformant d'anciennes fins en nouveaux moyens. Mary Douglas (1987 : 66-67) propose d'étendre la notion de bricolage au champ des institutions modernes.

\section{B I B L I O G R A P H IE}

ACHARD, Pierre

1982 « Au Nom du père. Ébauche du système anthroponymique français », Langage et Société 22 : $25-45$.

BALANDIER, Georges

1985 Le Détour. Paris, Fayard.

BERRY, Michel

1983 Une Technologie invisible. École Polytechnique, Centre de Recherche en Gestion.

BROWN, Roger \& Marguerite FORD

1964 " Address in American English », in Dell Hymes, ed., Language in Culture and Society. New York, Harper \& Row : 234-244.

BRown, Roger \& Albert GilmaN

1968 "The Pronouns of Power and Solidarity ", in J. A. Fischman, ed., Readings in the Sociology of Language. La Haye-Paris, Mouton : 252-275.

Delpeuch, Jean-Luc \& Anne Lauvergeon

1986 «Sur les Traces des dirigeants», Annales des Mines. Gérer et Comprendre 2 : 20-31.

1988 Sur les Traces des dirigeants. Paris, Calmann-Lévy.

Douglas, Mary

1987 How Institutions Think. London, Routledge \& Kegan Paul. (1986, Syracuse, NY, Syracuse University Press.)

Ethnographie des organisations. Actes du séminaire du Centre de Recherche en Gestion, École Polytechnique, 1984-1, 1984-2, 1985, 1986, 1987, 1988.

Fishman, J. A., ed.

1968 Readings in the Sociology of Language. La Haye-Paris, Mouton.

GoFFMAN, Erving

1973 La Mise en scène de la vie quotidienne. 1 : La Présentation de soi. Paris, Minuit.

Gomes DA Silva, José Carlos

1983 « Nous-mêmes, nous autres », L'Homme XXIII (3) : 55-80.

Guigo, Denis

1986 « Le Système de parenté tibétain », L'Ethnographie 98-99 : 71-117.

1987 «L'Empire du consensus », Annales des Mines. Gérer et Comprendre 6 : 19-26 ; 7 : 24-33.

Hall, Edward T.

1978 La Dimension cachée. Paris, Seuil (« Points »). (Éd. orig. 1966.) 
HÉRITIER, Françoise

1979 «Symbolique de l'inceste et de sa prohibition », in Michel IZARD \& Pierre SMITH:, eds., $L a$ Fonction symbolique. Paris, Gallimard (« Bibliothèque des Sciences humaines »): 209-243.

1981 L'Exercice de la parenté. Paris, Hautes Études, Gallimard/Seuil.

L'Homme XX (4), 1980 : Formes de nomination en Europe.

HYMES, Dell, ed.

1964 Language in Culture and Society. New York, Harper \& Row.

LEROI-GOURHAN, André

1968 « L’Expérience ethnologique », in Encyclopédie de la Pléiade. Ethnologie générale. Paris, Gallimard : 1816-1825.

LÉvi-STrauss, Claude

1958 Anthropologie structurale. Paris, Plon.

1985 La Pensée sauvage. Paris, Plon (" Agora »). (1

Niedermayer-TAHRI, Sigrid

1978 Les Appellatifs dans trois types d'institutions (l'hôpital psychiatrique, la prison, l'usine d'automobiles) et dans trois pays (en Allemagne, en France et en Angleterre), étude sociolinguistique comparative. Thèse de $3^{\mathrm{e}}$ cycle (non publiée), Université de Paris VII.

WINKIN, Yves

1981 La Nouvelle communication. Paris, Seuil (« Points »). 


\begin{abstract}
A B S T R A C T
Denis Guigo, How Colleagues Address Each Other in a Parisian Office. - A firm asked an anthropologist to do a study, and he saw this as an opportunity to examine how colleagues address each other. Do they use first or last names ? Do they say Mr. or Mrs. ? tu or vous ? The analysis of each term reveals a dynamic process of keeping the " right distance ", wherein age and sex differences as well as organizational principles (hierarchy, division of labor) have their part. Studying terms of address paves the way to an anthropological approach to firms : a firm is not an isolated entity, but specific dynamics - related, first of all, to differentiation- arise and develop within it.
\end{abstract}

\title{
ZUSAMMENFA S S N G
}

Denis Guigo, Anredeformen in einem Pariser Büro. - Ein Großunternehmen vertraut einem Ethnologen eine Untersuchung an. Dieser erblickt darin die Gelegenheit, unterschiedliche Anredeformen zu erfassen : Wendet man sich an einen Kollegen mit seinem Familiennamen oder seinem Vornamen ? Redet man ihn mit $d u$, Sie, Monsieur oder Madame an ? Die Analyse der verwendeten Terminologien läßt eine Dynamik der « guten Distanz » erkennen, in welcher Trennungen (nach Geschlecht und Alter) und organisatorische Prinzipien (Hierarchie, Aufgabenteilung) am Werk sind. Die Untersuchung der Anredeformen öffnet damit Wege zu einer ethnologischen Konstruktion des Forschungsgegenstands Unternehmen : Ein Unternehmen ist kein Isolat; vielmehr entstehen und entwickeln sich in ihm spezifische Funktionsweisen, die an erster Stelle auf das Ausüben einer Differenzierung verweisen.

\section{RES UMEN}

Denis Guigo, Términos de apelación en una oficina parisina. - Una gran empresa confía un estudio a un antropólogo que encuentra allí la oportunidad de relevar el vocabulario de apelación : ¿nos dirigimos a un colega por su nombre, o su apellido? ¿Le tratamos de tu, Usted, Señor o Señora? El análisis de la terminología empleada permite ver una dinámica de la « distancia apropiada » en la cual operan las diferencias de sexo y edad y los principios organizacionales (jerarquía, división de tareas). De esta manera, el estudio de los términos de apelación abre nuevas perspectivas para la investigación antropológica de las organizaciones : una empresa se considera como situada dentro de una sociedad, pero en su interior nacen y se desarrollan funcionamientos específicos que hacen a la diferenciación. (Trad. D.G.) 